\title{
Femtosecond optical reflectivity measurements of lattice-mediated spin repulsions in photoexcited $\mathrm{LaCoO}_{3}$ thin films
}

\author{
J. Bielecki, ${ }^{1,2, *}$ A. D. Rata, ${ }^{3, \dagger}$ and L. Börjesson ${ }^{1}$ \\ ${ }^{1}$ Department of Applied Physics, Chalmers University of Technology, SE-41296 Göteborg, Sweden \\ ${ }^{2}$ Department of Cell and Molecular Biology, Uppsala University, Box 596, SE-75124 Uppsala, Sweden \\ ${ }^{3}$ IFW Dresden, Institute for Metallic Materials, Helmholtzstraße 20, 01069 Dresden, Germany \\ (Received 9 November 2012; revised manuscript received 29 December 2013; published 21 January 2014)
}

\begin{abstract}
We present results on the temperature dependence of ultrafast electron and lattice dynamics, measured with pump-probe transient reflectivity experiments, of an epitaxially grown $\mathrm{LaCoO}_{3}$ thin film under tensile strain. Probing spin-polarized transitions into the antibonding $e_{g}$ band provides a measure of the low-spin fraction, both as a function of temperature and time after photoexcitation. It is observed that femtosecond laser pulses destabilize the constant low-spin fraction $(\sim 63 \%-64 \%)$ in equilibrium into a thermally activated state, driven by a subpicosecond change in spin gap $\Delta$. From the time evolution of the low-spin fraction, it is possible to disentangle the thermal and lattice contributions to the spin state. A lattice mediated spin repulsion, identified as the governing factor determining the equilibrium spin state in thin-film $\mathrm{LaCoO}_{3}$, is observed. These results suggests that time-resolved spectroscopy is a sensitive probe of the spin state in $\mathrm{LaCoO}_{3}$ thin films, with the potential to bring forward quantitative insight into the complicated interplay between structure and spin state in $\mathrm{LaCoO}_{3}$.
\end{abstract}

\section{INTRODUCTION}

The transition-metal perovskite $\mathrm{LaCoO}_{3}$ has attracted considerable attention as a consequence of its puzzling spin-state transitions [1-3]. In the ground state, $\mathrm{LaCoO}_{3}$ is a diamagnetic insulator with rhombohedral space group $R \overline{3} c$ and low-spin (LS) $d$-orbital occupancy $t_{2 g}^{6} g_{g}^{0}$. With increasing temperature the spin state gradually transforms towards a paramagnetic state, which has been discussed to be of either intermediate spin (IS) $t_{2 g}^{5} e_{g}^{1}(S=1)$ or high-spin (HS) $t_{2 g}^{4} e_{g}^{2}(S=2)$ character.

The driving mechanism behind the spin-state transition has been attributed to the sensitive interplay between the crystal field splitting $\Delta_{\mathrm{CF}}$, the intraionic exchange energy $\Delta_{U}$, and the $e_{g}$ bandwidth $W$, via the spin-gap $\Delta=\Delta_{\mathrm{CF}}-W / 2-\Delta_{U}$ found to be $\sim 200 \mathrm{~K}(17 \mathrm{meV})$ in magnitude. $\Delta$ allows a thermally depopulated low-spin fraction according to Fermi statistics $[4,5]$;

$$
n_{\mathrm{LS}}(T)=\frac{1}{1+Z \exp (-\Delta / T)},
$$

where $Z=(2 S+1) \eta$ is the combined spin and orbital degeneracy of the excited state.

It was realized early on by Goodenough $[2,3]$ that the ionic size difference between the small LS and large $\mathrm{HS} \mathrm{Co}^{3+}$ ions can stabilize a 1:1 LS-HS ratio in a checkerboard pattern, where cooperative lattice effects act as a spin repulsion (SR) that prohibits further HS occupation. However, the observed $n_{\mathrm{LS}}$ exhibit neither the simple activation according to Eq. (1) nor the 1:1 LS-HS ratio at elevated temperatures. Instead, lattice interactions have been discussed as affecting $n_{\mathrm{LS}}$, for example, in terms of an effective temperature-dependent spin

\footnotetext{
*johanb@xray.bmc.uu.se

${ }^{\dagger}$ Present address: Max Planck Institute for Chemical Physics of Solids, Nöthnitzerstraße 40, DE-01187 Dresden, Germany.
}

gap [6] or "negative cooperativity" [7]; where $\Delta$ increases with the number of $\mathrm{HS}$ sites, $n_{\mathrm{HS}}$, through an energy of mixing between LS and HS sites.

Recently, a ferromagnetic (FM) state with HS character even at low temperature was observed in epitaxially grown $\mathrm{LaCoO}_{3}$ thin films [8-15]. The HS character is closely connected to substrate induced strain; polycrystalline thin films grown on Si substrates do not exhibit an FM HS ground state $[8,11]$. Moreover, $\mathrm{x}$-ray absorption spectroscopy (XAS) complemented by atomic multiplet calculations indicates that epitaxial thin-film $\mathrm{LaCoO}_{3}$ grown on $\mathrm{SrTiO}_{3}$ (STO) have a constant LS fraction $n_{\mathrm{LS}}=0.64$ in the temperature range 10 $300 \mathrm{~K}[15,16]$, in contrast to bulk $\mathrm{LaCoO}_{3}$ as discussed above. The first experimental observations of a low-temperature HS state in strained $\mathrm{LaCoO}_{3}$ thin films were discussed in terms of an increase in orbital overlap as the epitaxial strain increases the $\mathrm{Co}-\mathrm{O}-\mathrm{Co}$ angles $[8,9,15]$. Calculations [17] and a recent XAS study [18] instead emphasize the structural deformations as the driving mechanism behind the substrate-dependent HS fraction.

Lattice interactions are thus expected to be an important factor in the realized spin states, both in thin-film and bulk $\mathrm{LaCoO}_{3}$. In this paper, we investigate the lattice contributions to $n_{\mathrm{LS}}$ in thin film $\mathrm{LaCoO}_{3}$ using femtosecond transient reflectivity measurements. Time-resolved pump-probe spectroscopy provides a powerful technique for directly investigating the electron, lattice, and spin dynamics on a subpicosecond time scale and has previously been used to temporally disentangle the origins of many unique properties of transition metal oxides [19-21]. Here, we show that $n_{\mathrm{LS}}$ can be measured by probing magneto-optical transitions in thin-film $\mathrm{LaCoO}_{3}$. It is observed that the underlying $n_{\mathrm{LS}}$ follows the purely thermal activation [Eq. (1)], while the time evolution of $n_{\mathrm{LS}}$ reveals a lattice mediated spin repulsion, as first proposed by Goodenough [2]. We argue that the SR is responsible for the deviations from a thermal spin population, both in the equilibrium and photoexcited states. 


\section{EXPERIMENTAL}

The $\mathrm{LaCoO}_{3}$ thin film with thickness of $100 \mathrm{~nm}$ was epitaxially and coherently grown on a STO substrate by pulsed laser deposition. The in-plane lattice parameter of the $\mathrm{LaCoO}_{3}$ film is identical with the substrate one, thus the $\mathrm{LaCoO}_{3}$ film is fully strained and exhibits a FM state below $85 \mathrm{~K}$. Above $85 \mathrm{~K}$, the ferromagnetic order disappears and a paramagnetic state is instead observed up to at least $300 \mathrm{~K}$. Details about the growth, structural, and magnetic characterization can be found elsewhere [11].

Time-resolved reflectivity measurements were performed at near normal incidence with a Ti:sapphire mode-locked amplifier with a $1-\mathrm{kHz}$ repetition rate and $150-\mathrm{fs}$ pulse duration. The $p$-polarized pump beam was set to $777 \mathrm{~nm}$ (1.6 $\mathrm{eV})$, while the probe beam, obtained from the $s$-polarized second harmonic signal from a $\beta$-BBO crystal, was fixed at the second harmonic wavelength $388 \mathrm{~nm}(3.2 \mathrm{eV})$. The pump and probe wavelengths were chosen to match the broad magneto-optical transitions centered at 1.5 and $3.1 \mathrm{eV}$, respectively [22]. The beam diameters at the sample surface were adjusted to 135 and $160 \mu \mathrm{m}$ for the probe and pump, respectively, giving a constant pump fluence $<1 \mathrm{~mJ} / \mathrm{cm}^{2}$, resulting in $\sim 0.03$ absorbed photons/Co site and a probe/pump fluence ratio of less than $1 / 100$.

\section{RESULTS}

Figure 1(a) shows the first 4 ps of the transient reflectivity normalized to the peak value at $300 \mathrm{~K}, d R_{\mathrm{N}}$, in the temperature interval $10-300 \mathrm{~K}$. The initial rise of $d R_{\mathrm{N}}$ centered around $0 \mathrm{ps}$ delay arises from the instantaneous hole-carrier excitation. After the initial photoexcitation, a fast decay from the thermalization of excited carriers is observed on the 100-fs time scale [23,24]. The amplitude of this decay is relatively weak at $300 \mathrm{~K}$ and increases rapidly below $100 \mathrm{~K}$, see the time-temperature 2D plot in Fig. 1(b).

As the minimum in $d R_{\mathrm{N}}$ is reached, a second process takes over, see Fig. 1(c) where delays up to $20 \mathrm{ps}$ are shown. This process is due to a combination of the nonthermal lattice relaxation (LR) previously seen in cobaltite systems [25] and the usual coherent acoustical phonons (CAPs) from its wavefront [26], propagating through the sample at the speed of sound. Notice also the lack of significant changes in the dynamics between 10-100 K, where mainly a translational offset due to increased strength of the subpicosecond decay is observed with decreasing temperature.

After $17 \mathrm{ps}$, the LR and CAPs reach the substrate as indicated by discontinuity at the broken line in Fig. 1(d) where $d R_{\mathrm{N}}$ up to $200 \mathrm{ps}$ is shown. The persistent oscillations show that the CAPs continue into the bulk of STO with a frequency of $113 \mathrm{GHz}$ as inferred from the power spectrum in the inset. We obtain a speed of sound $C_{\mathrm{LCO}}=5.9$ and $C_{\mathrm{STO}}=9.0 \mathrm{~km} / \mathrm{s}$, respectively.

The probe pulse at $3.2 \mathrm{eV}$ is in resonance with the spinpolarized and formally dipole-forbidden $t_{2 g} \rightarrow e_{g}^{*}$ transition centered at $3.1 \mathrm{eV}$, from $\mathrm{O} 2 p$ derived to $\mathrm{Co} d$ derived bands [22]. However, Co-O hybridization effects make this transition dipole-allowed [22], and the observed features in $d R_{\mathrm{N}}$ are thus sensitive to the density of empty $e_{g}^{*}$ states and,
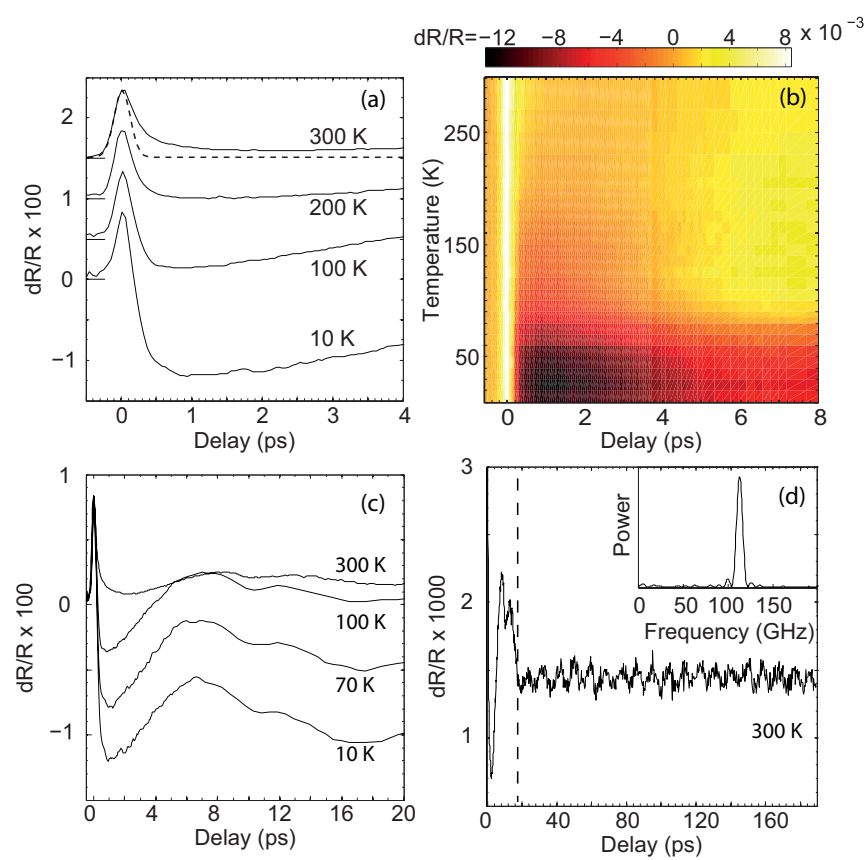

FIG. 1. (Color online) Transient reflectivity of a $\mathrm{LaCoO}_{3}$ thin film under tensile strain in the temperature range 10-300 K. Each transient is normalized to the $300 \mathrm{~K}$ peak value at $0 \mathrm{ps}$. (a) shows transients at $300,200,100$, and $10 \mathrm{~K}$. The broken line is the pump pulse autocorrelation and indicates the achievable time resolution. For clarity, each transient has been offset along the $y$ axis, with the respective zero-level indicated by the leftmost solid line. In (b), transients for all measured temperatures are collected in a 2D plot with $d R / R$ given by the color bar. (c) Transient reflectivity in the $0-20 \mathrm{ps}$ range. (d) $d R / R$ at $300 \mathrm{~K}$ for $0-200 \mathrm{ps}$. From the constant

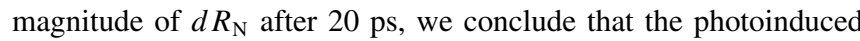
change in the spin state is long lived, returning to equilibrium as the holes and carriers recombine.

consequently, to $n_{\mathrm{LS}}$, which in its purest form follows the thermally activated behavior in Eq. (1).

To visualize the time and temperature evolution of $n_{\mathrm{LS}}(T, \tau)$, we make vertical cuts in Fig. 1(b) at different delays $\tau$. The cut taken at $\tau=500$ fs [see the red circles in Fig. 2(a)] is fitted well to Eq. (1), both with a HS $S=2$ model where $\Delta=200 \pm 10 \mathrm{~K}$ and $Z=5$ (solid line) and with an IS $S=1$ model, where $\Delta=175 \pm 8 \mathrm{~K}$ and $Z=3$ (broken line), and it is from these fits that the numerical $n_{\mathrm{LS}}(T, \tau)$ values are extracted. Based on previous experimental $[15,16]$ and computational [14] works, we adopt the HS interpretation in the following discussions. The excellent fit of Eq. (1) to the data is the first verification that $d R_{\mathrm{N}}$ indeed contain information about $n_{\mathrm{LS}}$. At larger $\tau$, the thermal evolution of $n_{\mathrm{LS}}$ begins to deviate from the thermally activated state. Here, a rapid increase of $n_{\mathrm{LS}}$ towards the equilibrium low-spin fraction $n_{\text {eq }}=n_{\mathrm{LS}}(T, \infty)$ is observed for $T \geqslant 100 \mathrm{~K}$, while below 100 $\mathrm{K}$, we observe an $n_{\mathrm{LS}}$ independent on $\tau$, see Fig. 2(a). The static $n_{\mathrm{LS}}$ below $100 \mathrm{~K}$ can also be observed directly in the experimental $d R_{\mathrm{N}}$ data as the previously mentioned invariant shape below $100 \mathrm{~K}$ seen in Fig. 1(c).

The increase in $n_{\mathrm{LS}}$ as a function of delay at $300 \mathrm{~K}$ is highlighted in Fig. 2(b) where $n_{\mathrm{LS}}$ is plotted as a function of $\tau$, 

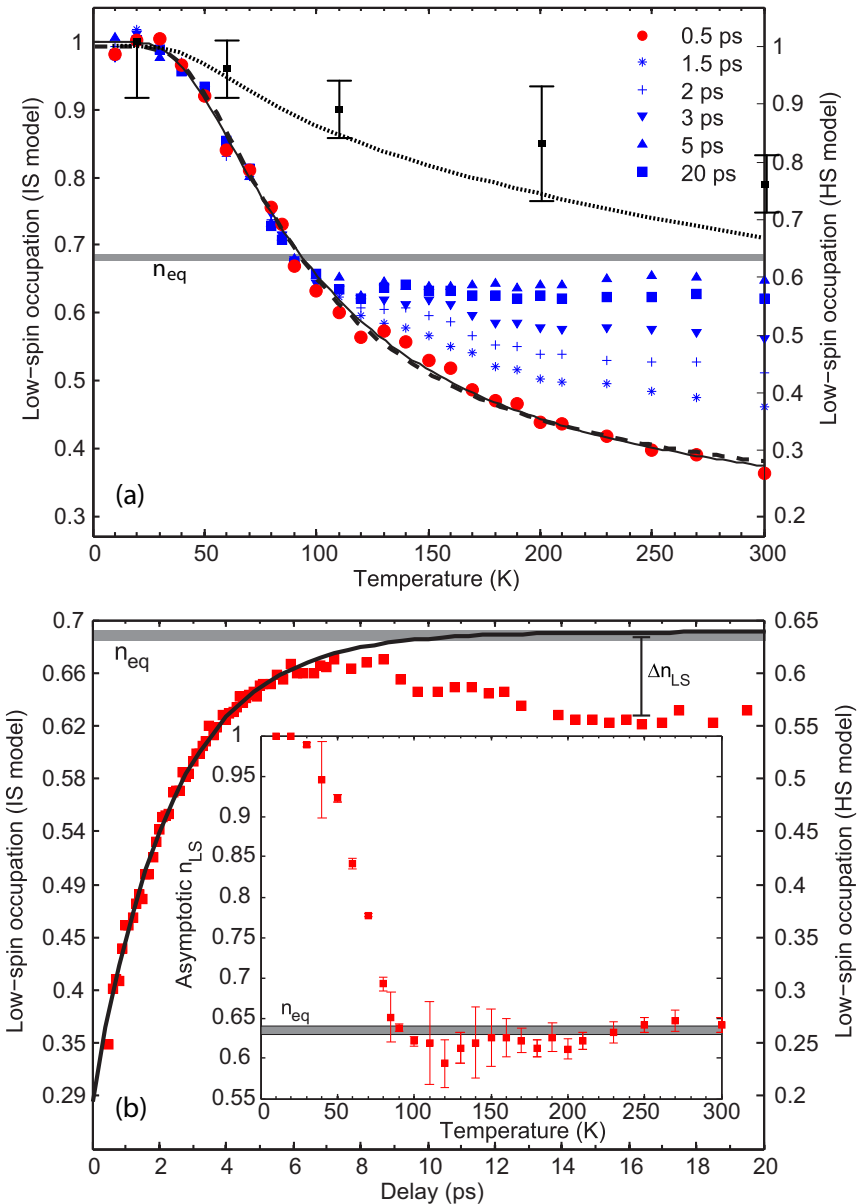

FIG. 2. (Color online) (a) The temperature and time-dependent low-spin fraction $n_{\mathrm{LS}}(T, \tau)$, extracted from the measured $d R / R$ as explained in the text, for six different time delays. Bulk data from Ref. [6] are shown with the black squares and error bars together with the calculated $n_{\text {LS }}$ from Ref. [30] (dotted line). (b) A vertical cut at $300 \mathrm{~K}$, showing the complete time evolution of $n_{\mathrm{LS}}(300 \mathrm{~K}, \tau)$ (red squares) after subtraction of oscillations from CAPs. An exponential (solid line) provides a good fit to the initial increase of $n_{\mathrm{LS}}(300 \mathrm{~K}, \tau)$. In the inset, the electron-lattice equilibrium LS fraction obtained from the exponential fit is shown for all temperatures. The HS model gives an average of $62.5 \%$, in agreement with the previously estimated $n_{\text {eq }}=0.63-0.64$ (shaded areas) $[15,16]$.

after the removal of an oscillatory component from the LR and CAPs [26]. $n_{\mathrm{LS}}$ increases rapidly between 0.5 and 8 ps before a slow decrease sets in. The rapid increase can be described with a simple exponential that asymptotically reaches the equilibrium value $n_{\mathrm{LS}}=0.64$ with time constant $\tau_{\mathrm{e}-1}=2.8 \mathrm{ps}$, typical for electron-lattice (e-1) relaxations [23,24,27-29]. The decrease in $n_{\mathrm{LS}}$ after $8 \mathrm{ps}$ is attributed to the LR process discussed above [25].

In the inset of Fig. 2(b), the asymptotic $n_{\mathrm{LS}}$, with error bars indicating a $95 \%$ confidence interval, obtained in this way is plotted for all temperatures. Above $90 \mathrm{~K}, n_{\mathrm{LS}}$ varies between $60 \%$ and $64 \%$ and these variations can at least partly be assigned to uncertainties arising from the exponential fitting and removal of the oscillatory CAP signal. The fact that we obtain a constant $n_{\text {eq }}$ with the same value as reported elsewhere $[15,16]$ provides a nontrivial consistency check of our results and interpretation of the pump-probe signal. However, it should be noted that it is in general not possible to extract $n_{\mathrm{LS}}(T, \tau)$ from $d R_{\mathrm{N}}(T, \tau)$ without additional constraints. In particular, we expect it to be difficult to extend the methodology used here to interpret analogous experiments performed on bulk $\mathrm{LaCoO}_{3}$. For thin-film $\mathrm{LaCoO}_{3}$, the possibility to extract the absolute $n_{\mathrm{LS}}(T, \tau)$ hinges on the fact that $n_{\text {eq }}$ has been reported to be constant in the $10-300-\mathrm{K}$ temperature range $[15,16]$, although we stress that the actual numerical value of $n_{\text {eq }}$ was not used as input in obtaining $n_{\mathrm{LS}}(T, \tau)$.

\section{DISCUSSION}

Our observations can be explained as follows. The photoexcitation with 1.6-eV photons is in resonance with the magneto-optical transition at $1.5 \mathrm{eV}$ and excites electrons into the Co derived $e_{g}^{*}$ band [22,25], creating hole-carrier pairs with a quasistable concentration during the remainder of our measurements [24]. The observed effect is a pump pulse intensity dependent increase of $\Delta$, leading to a ferromagnet to diamagnet collapse of the equilibrium $n_{\mathrm{LS}}$ into a distribution following a purely thermal activation [red circles in Fig. 2(a)]. As this process occurs on a subpicosecond time scale, we assign this increase of $\Delta$ to an electronic process where the creation of holes decreases the orbital overlap and $\mathrm{O} 2 p-\mathrm{Co}$ $3 d$ bandwidth.

Below $100 \mathrm{~K}, n_{\mathrm{LS}}$ follows an essentially static thermal occupation that relaxes back to $n_{\mathrm{eq}}$ as the equilibrium $\Delta$ is recovered on the hole-carrier recombination time scale. Above $100 \mathrm{~K}, n_{\mathrm{LS}}$ increases towards $n_{\mathrm{eq}}$ on the e- 1 relaxation time scale and we interpret this as a lattice mediated SR, through cooperative oxygen displacements to accommodate the large $\mathrm{HS} \mathrm{Co}^{3+}$ ions. From the abrupt crossover from thermally excited to SR saturated $n_{\mathrm{LS}}$ at $100 \mathrm{~K}$, we deduce that the effective strength of the SR is zero above a critical $n_{\mathrm{LS}}$, below which the SR becomes strong enough to completely negate further thermal LS depopulation in the temperature range investigated here. This argument is summarized in Fig. 3.

After e-1 relaxation but before carrier-hole recombination, the observed spin states are remarkably similar to the idealized model describing bulk $\mathrm{LaCoO}_{3}$ proposed by Goodenough, which incorporates a thermally activated $n_{\mathrm{LS}}$ until the SR stabilizes a checkerboard $n_{\mathrm{LS}}=0.5$ arrangement $[2,3,30]$. The discrepancy between the predicted $n_{\mathrm{LS}}=0.5$ and the observed $n_{\mathrm{LS}}=0.64$ implies that it is necessary to take into account collective electron-lattice interactions beyond the nearest-neighbor approximation to fully understand the SR and we expect these interactions to be sensitive to the detailed crystal structure and thus strain.

Indeed, a clear dependence of the SR on the strain state is observed in Fig. 2(b) from the decrease $\Delta n_{\mathrm{LS}}$ in $n_{\mathrm{LS}}$ above 8 ps due to the increased strain from the LR, which temporarily decreases the lattice interactions by acting as negative pressure [25]. From a comparison between the 0.5 and 20-ps LS distributions below $100 \mathrm{~K}$ in Fig. 2(a), it can be seen that $\Delta$ is not affected by the LR, i.e., the SR is decoupled from the spin gap magnitude.

The equilibrium thin-film state can now be understood as having a suppressed $\Delta$ due to strain induced lattice 

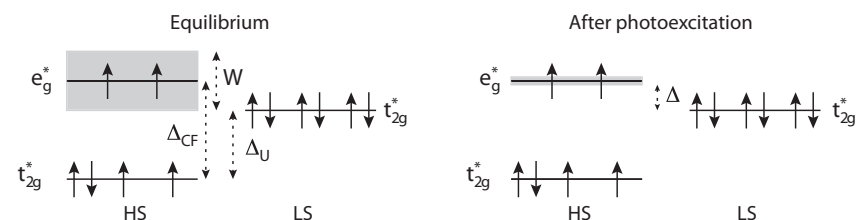

LS
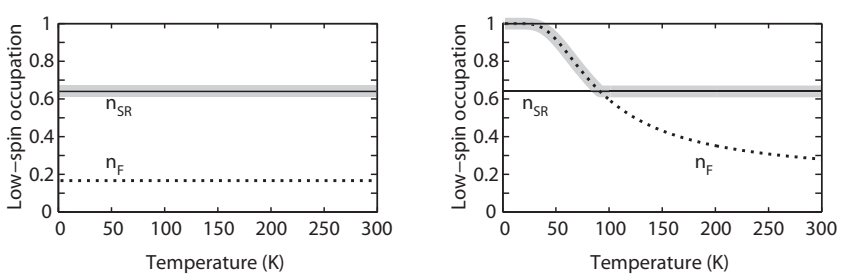

FIG. 3. Schematic over the effect of photoexcitation on $n_{\text {LS }}$. The upper left panel shows the relative energies [31] in a hypothetical equilibrium state where $\Delta=0$, including the crystal-field splitting $\Delta_{\mathrm{CF}}$, the intra-atomic exchange energy $\Delta_{U}$ and the $p-d$ bandwidth $W$. The resulting $n_{\mathrm{LS}}$, shown in the lower left panel (shaded area), is constant and constrained by the SR (solid line) as opposed to the Fermi distribution $n_{F}$ (broken line). The experimentally observed $n_{\text {eq }}=0.64$ above $10 \mathrm{~K}$ is consistent with $\Delta \leqslant 20 \mathrm{~K}$. Photoexcitation (upper right panel) decrease $W$ to give an increased $\Delta$. In this case, $n_{\mathrm{LS}}$ follows the Fermi distribution $n_{F}$ until the low-spin fraction given by the SR is reached (lower right panel). For clarity, both the magnitude and change in $W$ is greatly exaggerated.

deformations, whereby the SR determines $n_{\mathrm{LS}}$ down to at least $10 \mathrm{~K}$. This constrains $\Delta$ to $\leqslant 20 \mathrm{~K}$. Because the SR is very sensitive to the structural properties of the thin film, different $n_{\mathrm{LS}}$ and consequently magnetization strengths are observed on different substrates [8,11]. Further, the constant SR observed in the thin film could be explained by a quenching of the anomalous lattice expansion that couples to the spin state in bulk $\mathrm{LaCoO}_{3}[32,33]$ by epitaxial adhesion to the substrate. This explanation of the variations in magnetization on different substrates in terms of the SR is in accord with the recent XAFS study by Sterbinsky et al. [18] where it was argued that structural distortions are the important parameters for stabilizing the HS phase rather than the strength of the orbital hybridization.
Our conclusions supplement previous interpretations of the spin-state evolution in bulk $\mathrm{LaCoO}_{3}$, where a temperature dependent spin gap $\Delta(T)$, influenced by lattice relaxations, was used to explain the deviations from a thermally excited spin fraction [6,7]. While temperature induced variations of the lattice parameters are expected to affect $\Delta$ via the $p-d$ bandwidth and $\mathrm{Co}-\mathrm{O}$ bond length [31], we here demonstrate the decoupling of $\Delta(T)$ and the significant contribution to $n_{\mathrm{LS}}$ from the SR. As shown in the strain-induced low-spin decrease $\Delta n_{\text {LS }}$ [see Fig. 2(b)], the SR strength should also be expected to vary with strain and temperature. This suggests that $n_{\mathrm{LS}}$ in bulk $\mathrm{LaCoO}_{3}$ [shown in Fig. 2(a), squares with error bars [6] and dotted line [30]] is determined by a temperature dependent interplay between $\Delta$ and the SR.

\section{SUMMARY AND CONCLUSIONS}

In summary, we have presented time-resolved pumpprobe measurements on $\mathrm{LaCoO}_{3}$, where irradiation with a femtosecond 1.6-eV laser pulse causes an increase in $\Delta$, which initiates a ferromagnet to diamagnet transition occurring at the subpicosecond time scale. Probing the magneto-optical transition at $3.2 \mathrm{eV}$, we are able to disentangle the thermal from the lattice contributions to $n_{\mathrm{LS}}$ and measure the ultrafast spin-state evolution.

We observe a lattice mediated spin repulsion and argue that this effect determines the value of the $n_{\mathrm{LS}}$ and, consequently, the ferromagnetic moment, in tensile strained $\mathrm{LaCoO}_{3}$ thin films. Even though lattice interactions have been widely suggested as affecting $n_{\mathrm{HS}}$ in $\mathrm{LaCoO}_{3}[2,3,6,30,32]$, to our best knowledge, this is the first time these effects have been observed directly. Further time-resolved studies are needed to provide a clear understanding of the spin repulsion mechanism, which could assist in unraveling the spin-state transition in $\mathrm{LaCoO}_{3}$.

\section{ACKNOWLEDGMENTS}

We are grateful to Ezio Zanghellini for valuable discussions and technical assistance. JB and LB acknowledge the generous financial support from the Swedish Research Council (VR).
[1] M. Imada, A. Fujimori, and Y. Tokura, Rev. Mod. Phys. 70, 1039 (1998).

[2] J. B. Goodenough, J. Phys. Chem. Solids 6, 287 (1958).

[3] M. A. Senariz-Rodriguez and J. B. Goodenough, J. Solid State Chem. 116, 224 (1995).

[4] S. W. Biernacki, Phys. Rev. B 74, 184420 (2006).

[5] A. Ishikawa, J. Nohara, and S. Sugai, Phys. Rev. Lett. 93, 136401 (2004).

[6] M. W. Haverkort, Z. Hu, J. C. Cezar, T. Burnus, H. Hartmann, M. Reuther, C. Zobel, T. Lorenz, A. Tanaka, N. B. Brookes, H. H. Hsieh, H. J. Lin, C. T. Chen, and L. H. Tjeng, Phys. Rev. Lett. 97, 176405 (2006).

[7] T. Kyômen, Y. Asaka, and M. Itoh, Phys. Rev. B 67, 144424 (2003).
[8] D. Fuchs, C. Pinta, T. Schwarz, P. Schweiss, P. Nagel, S. Schuppler, R. Schneider, M. Merz, G. Roth, and H. von Loehneysen, Phys. Rev. B 75, 144402 (2007).

[9] D. Fuchs, E. Arac, C. Pinta, S. Schuppler, R. Schneider, and H. von Loehneysen, Phys. Rev. B 77, 014434 (2008).

[10] V. V. Mehta, M. Liberati, F. J. Wong, R. V. Chopdekar, E. Arenholz, and Y. Suzuki, J. Appl. Phys. 105, 07 E503 (2009).

[11] A. D. Rata, A. Herklotz, L. Schultz, and K. Dorr, EPJ B 76, 215 (2010).

[12] J. W. Freeland, J. X. Ma, and J. Shi, Appl. Phys. Lett. 93, 212501 (2008).

[13] A. Herklotz, A. D. Rata, L. Schultz, and K. Dorr, Phys. Rev. B 79, 092409 (2009).

[14] H. Hsu, P. Blaha, and R. M. Wentzcovitch, Phys. Rev. B 85, 140404 (2012). 
[15] M. Merz, P. Nagel, C. Pinta, A. Samartsev, H. v Loehneysen, M. Wissinger, S. Uebe, A. Assmann, D. Fuchs, and S. Schuppler, Phys. Rev. B 82, 174416 (2010).

[16] C. Pinta, D. Fuchs, M. Merz, M. Wissinger, E. Arac, H. Von Loehneysen, A. Samartsev, P. Nagel, and S. Schuppler, Phys. Rev. B 78, 174402 (2008).

[17] J. M. Rondinelli and N. A. Spaldin, Phys. Rev. B 79, 054409 (2009).

[18] G. E. Sterbinsky, P. J. Ryan, J. W. Kim, E. Karapetrova, J. X. Ma, J. Shi, and J. C. Woicik, Phys. Rev. B 85, 020403 (2012).

[19] C. Kübler, H. Ehrke, R. Huber, R. Lopez, A. Halabica, R. F. Haglund, Jr., and A. Leitenstorfer, Phys. Rev. Lett. 99, 116401 (2007).

[20] M. Rini, R. Tobey, N. Dean, J. Itatani, Y. Tomioka, Y. Tokura, R. W. Schoenlein, and A. Cavalleri, Nature (London) 449, 72 (2007).

[21] S. Dal Conte, C. Giannetti, G. Coslovich, F. Cilento, D. Bossini, T. Abebaw, F. Banfi, G. Ferrini, H. Eisaki, M. Greven, A. Damascelli, D. van der Marel, and F. Parmigiani, Science 335, 1600 (2012).

[22] S. Yamaguchi, Y. Okimoto, K. Ishibashi, and Y. Tokura, Phys. Rev. B 58, 6862 (1998).
[23] A. J. Sabbah and D. M. Riffe, Phys. Rev. B 66, 165217 (2002).

[24] S. Sundaram and E. Mazur, Nat. Mater. 1, 217 (2002).

[25] Y. Okimoto, X. Peng, M. Tamura, T. Morita, K. Onda, T. Ishikawa, S. Koshihara, N. Todoroki, T. Kyômen, and M. Itoh, Phys. Rev. Lett. 103, 027402 (2009).

[26] C. Thomsen, H. T. Grahn, H. J. Maris, and J. Tauc, Phys. Rev. B 34, 4129 (1986).

[27] R. D. Averitt, A. I. Lobad, C. Kwon, S. A. Trugman, V. K. Thorsmolle, and A. J. Taylor, Phys. Rev. Lett. 87, 017401 (2001).

[28] T. Ogasawara, K. Tobe, T. Kimura, H. Okamoto, and Y. Tokura, J. Phys. Soc. Jpn. 71, 2380 (2002).

[29] J. Bielecki, R. Rauer, E. Zanghellini, R. Gunnarsson, K. Dörr, and L. Börjesson, Phys. Rev. B 81, 064434 (2010).

[30] T. Kyômen, Y. Asaka, and M. Itoh, Phys. Rev. B 71, 024418 (2005).

[31] J. S. Zhou, J. Q. Yan, and J. B. Goodenough, Phys. Rev. B 71, R220103 (2005).

[32] S. Murata, S. Isida, M. Suzuki, Y. Kobayashi, K. Asai, and K. Kohn, Physica B 263, 647 (1999).

[33] C. Zobel, M. Kriener, D. Bruns, J. Baier, M. Gruninger, T. Lorenz, P. Reutler, and A. Revcolevschi, Phys. Rev. B 66, 020402 (2002). 